\title{
Insomnia induced brief manic-psychotic episodes
}

\begin{abstract}
The present article explores a novel psychiatric presentation triggered by insomnia. Specifically, the authors describe an acute pathology characterized by brief manic-psychosis and first episodes of psychosis or hypomania in people who suffer from prolonged sleep deprivation and insomnia. The presentation recedes after physiological sleep is restored. Although it was expected that tiredness and exhaustion would fire on the sleep center by promoting physiological sleep, this does not occur at all times. The authors of the current research work in a psychiatric setting with the general adult non-forensic population. During the recent years, there has been an increase of admissions of patients presenting with severe sleep deprivation, insomnia, and brief manic-psychotic episodes that subside once proper sleep has been restored. In the current communication, the authors discuss the neurophysiological and psychiatric aspects that link sleep to mental illnesses and insomnia to brief psychotic episodes. The current study reports on prototypical case studies which presented with the syndrome together with the diagnostic and clinical evidence for considering it as a distinct psychiatric pathology here named Insomnia Induced Brief Manic-Psychotic Episodes.
\end{abstract}

Keywords: insomnia, brief psychotic episode, mania, neurophysiology, mental illnesses
Volume 2 Issue 2 - 2018

\author{
Carlo Lazzari,' Ahmed Shoka,' Basavaraja \\ Papanna,' Marco Rabottini' ${ }^{2}$ \\ 'Essex Partnership University Foundation Trust, United Kingdom \\ ${ }^{2}$ Center for the Study of Sleep Disorders, Italy
}

Correspondence: Carlo Lazzari, Kingswood Centre, Turner Rd, Colchester, United Kingdom, Tel +0044 74947987 3I, Email carlolazzari2015@gmail.com

Received: February 07, 2018 | Published: March 07, 2018
Abbreviations: IIBMPE, insomnia induced brief manicpsychotic episodes; HTPA, hypothalamic pituitary adrenal axis

\section{Introduction}

Sleep hygiene and promotion of sleep have always been the focal concern of psychiatrists for their patients. The impact of sleep disorders and insomnia has also been recognized as central to the well-being of patients with mental illnesses. In psychiatric settings, insomnia usually presents as an early (difficulty in falling asleep) or late (early awakening) sleep difficulty till the most severe lack of sleep for days. However, although present in most of the psychiatric illnesses, insomnia, either pharmacologically induced (e.g., excitatory substances of abuse), physically produced (e.g., chronic pain), or mentally caused (e.g., obsessive ruminations), can trigger psychiatric relapses or first episodes of brief psychotic episodes.

Merriam-Webster Dictionary Online defines insomnia as "prolonged and usually abnormal inability to get enough sleep". Prolonged insomnia is a stressful condition. As a consequence, after stress, there is an increased production of adrenocorticotropin hormone $(\mathrm{ACTH})$ by the anterior pituitary gland ${ }^{2}$ with increased autonomic activity. ${ }^{3}$ The Insomnia Induced Brief Manic-Psychotic Episodes (IIBMPE) described in the current communication affect healthy people otherwise with a negative history of mental and behavioural disorders, and can be considered as a stressful condition. Several pathogenic hypotheses are postulated for the IIBMPE and its symptoms by the authors of the current study. For instance, the hyper arousal theory suggests that people who chronically ruminate about their sleep problem are more inclined to suffer from insomnia. ${ }^{4}$ A study based on self-reports identified a prevalence of $26 \%$ of insomnia in patients reporting first psychotic episodes in psychiatric care. ${ }^{5}$ The authors of the current research also recognized problematic impairment in performance of professionals who start to suffer from insomnia, including technical errors with reduction of fine motor skills and concentration. ${ }^{6}$ The current communication refers to clinical cases of persons who present to psychiatric hospitals with brief and first episodes of mixed mania and psychosis and who show complete remission after proper sleep, and sedative medications are started.
The assumption of the presence of a distinct psychiatric pathology entirely triggered by primary or secondary insomnia started by observing cases of brief psychotic episodes with manic features in college students. In fact, those students who later presented with IIBMPE reported reduced hours of sleep to increase their amount of study especially in the prediction of forthcoming exams. The presentation of IIBMPE into adult psychiatric ward was with brief psychotic disorders or frank psychotic mania with agitation, disinhibition, and paranoia. Milder forms of hypomania ensued for shorter periods of sleep deprivation and insomnia, usually comprising one or two nights of interrupted sleep in professionals working night shifts. ${ }^{6}$ However, the authors of the current study observed that for more extended periods of insomnia, subjectively described by affected people as a prolonged reduction or lack of physiological sleep, consequences were more serious hence triggering brief manic psychotic episodes.

The authors of the current research observed that the window period to trigger Insomnia Induced Brief Manic-Psychotic Episodes (IIBMPE) usually consists from a minimum of two or three nights of insomnia to up to seven or more nights subjectively reported as insomnia or lack of (proper) sleep. As the accounts collected by the authors were mostly subjective, collateral (family, friends), and from persons presenting with IIBMPE, there was no objective measurement of the degree of 'insomnia.' Nonetheless, subjective descriptions like 'lack of sleep, 'lack of proper sleep,' 'missing sleep,' together with psychiatric nurses' observations of patients being awake most of the nights were areas of serious concern. In the most common forms, the IIBMPE presents with grandiose delusions and the subjective feeling of not needing sleep and rest. Also, the person with IIBMPE believes of being capable of tackling any task. This presentation is accompanied by the reduction of critical thinking and insight into the subjective distress with the risk of further deterioration and impaired cognitive, behavioural, and functional skills.

The authors of the current communication created a rating scale to assess the degree of severity of IIBMPE (Table 1). Sadly, IIBMPE can also affect skilled professionals performing dangerous tasks. The authors believe that this occurrence should generate employers' 
concerns on the possible risk to self and others posed by people who start to lack hours of proper sleep like, for instance, when doctors, nurses, or airplane pilots are affected by IIBMPE. A vicious circle ensues where people who reported prolonged lack of sleep also felt that the need to sleep was less than required. At the same time, they described as satisfactory only two or three hours sleep in the twentyfour hours. However, the authors observed that once a proper sleep restarts, either pharmacologically induced or by stopping triggering causes for insomnia, the IIBMPE tended to subside while fully recovered patients can go back to healthy life practically symptoms free.

The authors of the current study hypothesized neurophysiological mechanisms for the IIBMPE, like a progressive reduction of the serotoninergic release with an increased noradrenergic-dopaminergic pressure on the sleep center. The authors of the current communication also suggest that after a few days of insomnia, the serotoninergic depletion is paralleled by increased activation of the hypothalamicpituitary-adrenal axis (HTPA axis) with consequent impairment of the cortisol release. However, this hypothesis needs experimental confirmation. Activation of HTPA axis manifests with increased autonomic activation ${ }^{3}$ like increased mean heart rate and blood pressure and other peripheral signs of the over-activation of the HTPA axis.

Consistent with these findings, the authors of the current study tried to restore the regular route to impaired sleep drive with cautious psychopharmacology. Mostly used were anti-histamine drugs having an affinity for benzodiazepine brain receptors like promethazine?. Other medications can be equally important, like all the class of benzodiazepines for a short course. However, people affected by IIBMPE might already have tried them and might have some resistance to benzodiazepine therapeutic effect. Furthermore, sedative antidepressants like trazodone can also be tried in the lower dosages to avoid that the antidepressant effect might reduce the sleep drive instead of improving it. In most serious cases, a small dosage of sedating antipsychotics like quetiapine or haloperidol was found to be beneficial when the clinicians suspected an organic basis for presentation or high anxious components for the disruption of sleep. It is notable that the elderly population might be very sensitive to acute infections (e.g., urinary, chest, etc.) that can generate an agitated delirium thus causing prolonged insomnia, confusion, and IIBMPE.

Moreover, the authors of the current communication observed that insomnia could occur in any form of sub-acute or chronic pain, or incorrect timing of psychotropic medications (e.g., antidepressants prescribed at night time). There are other causes of insomnia reported by literature like the constant use of caffeine based substances, ${ }^{7}$ bacterial infections and antibiotics, ${ }^{8}$ and multi-vitamins. ${ }^{9}$ Confirmation diagnosis arrives when the clinical and mental presentation of IIBMPE discontinues entirely after patients have undertaken an ordinary course of physiological sleep.

\section{Case studies}

The following section will illustrate three prototypical cases of IIBMPE which came under the authors' attention in their clinical practice. In order to arrive at articulated assessment tools, the authors met during several focus groups, and symptoms for possible IIBMPE were discussed as deriving from case studies. A qualitative analysis served in the generation of codes and items for the assessment questionnaire (Table 1). This last is a four-item questionnaire with three degrees of severity of IIBMPE symptoms reported on a threeitem Likert scale ranging from low (' 1 ') to maximum severity (' 3 '). The items of the IIBMPE assessment scale are, 1) behavioural components of IIBMPE with HPTA axis activation like irritability, restlessness, high pulse rate, etc., 2) cognitive aspects of IIBMPE, like reduced concentration, altered fine motor coordination, procedural errors, etc., 3) psychiatric symptoms including hypomania, irritability, restlessness, or clear mania and psychosis, etc., and 4) physical symptoms including activation of HTPA axis. The clinical diagnosis of IIBMPE was confirmed when the presentation matched all the following necessary parameters (NP) and some of the desirable parameters (DP). These parameters were: 1) negative past psychiatric history (NP), 2) full recovery from symptoms once sleep pattern is restored (NP), 3) negative brain magnetic resonance and electroencephalography (NP), 4) negative family history for major psychoses or affective disorders (DP); 5) absence of the recent abuse of excitatory (e.g., caffeine) (DP) and illicit excitatory drugs (NP) although moderate use might be found especially for caffeine, 6) absence of major health conditions (DP) which included altered thyroid function, uncontrolled diabetes, hypertension, infections, etc., 7) absence of medication which can alter sleep (DP) like, for instance, night prescribed antibiotics or vitamins. The following are three representative cases of IIBMPE.

\section{Case no. I}

This was the case of a 24-year-old female university student. She was admitted to a psychiatric ward with a confusional state, with restlessness, pressure of speech, flight of ideas, and grandiose delusions of being able to cure people just by touching them. On retrospective and collateral history it emerged that this young lady came to the United Kingdom as an international student and was very ambitious with a desire not to fail in her university exams. However, shortly before admission, she started to miss some rest and decided not to sleep at night to catch up with her study and to perform well at the university. Her friends started to notice bizarre behaviors. Therefore, they referred her to the local health unit which, finally, decided for admission to a psychiatric hospital. Her physical parameters were within normal limits. During admission, she was prescribed a short course of benzodiazepines and antipsychotics which eventually stopped the symptoms. Retrospective history reported by this patient evidenced progressive and voluntary reduction of sleep with probably three or more nights of insomnia when the hypomanic presentation started to occur. On the IIBMPE assessment scale, she scored 12, this score placing her in the severe-degree spectrum.

\section{Case no. 2}

This was the case of a 34-year-old man working for a local transport agency. He was admitted to a psychiatric ward with manifest manic symptoms, increased irritability, suspiciousness, pressure of speech but not flight of ideas. On the admission history, it emerged that due to conflicts and stress in his organization, this man started with minimal insomnia up to missing more than three nights of sleep. At admission, there was no restlessness. Physical parameters were all within normal limits. Past psychiatric history and family history of mental illnesses were negative. Similarly, in this case, a short course (three to five days) of benzodiazepines and antipsychotics halted the symptoms and increased physiological sleep. After three days, he fully recovered. He reported that obsessive ruminations about work bullying caused progressive insomnia that he tried to cure by increasing smoking and coffee at night time. He also spent increasing hours by surfing the 
Internet in search of jobs vacancies. This behavior became obsessive to the point that he started to miss more than 2 nights of sleep. On the
IIBMPE assessment scale, he scored 8, this score placing him in the moderate-degree spectrum.

Table I The stages of IIBMPE with scoring where the presence of each symptom accounts from I point to 3 points. The scale ranges from a minimum of I (low severity) to 8 (mild severity) to a final maximum of 12 (maximum severity) in IIBMPE severity of symptoms

\begin{tabular}{|c|c|c|c|c|c|c|}
\hline $\begin{array}{l}\text { IIBMPE } \\
\text { symptoms }\end{array}$ & $\begin{array}{l}\text { From one to } \\
\text { two nights of } \\
\text { insomnia }\end{array}$ & $\begin{array}{l}\text { Severity } \\
\text { Scoring }\end{array}$ & $\begin{array}{l}\text { From two to three } \\
\text { nights of insomnia }\end{array}$ & $\begin{array}{l}\text { Severity } \\
\text { Scoring }\end{array}$ & $\begin{array}{l}\text { More than three nights of } \\
\text { insomnia }\end{array}$ & $\begin{array}{l}\text { Severity } \\
\text { Scoring }\end{array}$ \\
\hline $\begin{array}{l}\text { Behavioral } \\
\text { components } \\
\text { and HTPA axis } \\
\text { activation }\end{array}$ & Slight irritability & I & $\begin{array}{l}\text { Increased irritability, } \\
\text { anger outbursts, and } \\
\text { conflict with others. }\end{array}$ & 2 & $\begin{array}{l}\text { Clear agitations with restlessness, } \\
\text { hypertension, fast heart rate, and } \\
\text { altered glucose metabolism. }\end{array}$ & 3 \\
\hline $\begin{array}{l}\text { Cognitive } \\
\text { aspects }\end{array}$ & $\begin{array}{c}\text { Reduced } \\
\text { concentration }\end{array}$ & I & $\begin{array}{l}\text { Minor cognitive } \\
\text { impairment } \\
\text { with omissions, } \\
\text { generalizations, and } \\
\text { altered judgment } \\
\text { in differences and } \\
\text { similarities. Minor } \\
\text { incidents in the use of } \\
\text { fine motor skills. }\end{array}$ & 2 & $\begin{array}{l}\text { Gross cognitive impairment, loss of } \\
\text { concentration and fine movement } \\
\text { coordination. Serious incidents, } \\
\text { like car accidents, or omissions } \\
\text { of basic skills in the own routine. } \\
\text { Professional errors. }\end{array}$ & 3 \\
\hline $\begin{array}{l}\text { Psychiatric } \\
\text { symptoms }\end{array}$ & $\begin{array}{l}\text { Hypomania, } \\
\text { paranoia. }\end{array}$ & I & $\begin{array}{l}\text { Mania, increased } \\
\text { suspiciousness, } \\
\text { overvalued ideas, } \\
\text { grandiose delusions, } \\
\text { paranoia. }\end{array}$ & 2 & $\begin{array}{l}\text { Frank manic psychosis, brief } \\
\text { paranoid psychosis, brief } \\
\text { psychotic episodes, first psychotic } \\
\text { presentation. Grandiose delusions } \\
\text { with heightened mood, reduced } \\
\text { critical thinking, unrealistic or } \\
\text { multiple plans, with increased } \\
\text { mystic thoughts. Manic-psychosis } \\
\text { with suspiciousness and grandiosity. }\end{array}$ & 3 \\
\hline $\begin{array}{l}\text { Physical } \\
\text { symptoms of } \\
\text { IIBMPE }\end{array}$ & $\begin{array}{l}\text { Minor alterations } \\
\text { from activation } \\
\text { of HTPA axis, } \\
\text { e.g., increased } \\
\text { heart rate, a } \\
\text { slight increase in } \\
\text { diastolic blood } \\
\text { pressure, minor } \\
\text { glucose metabolism } \\
\text { alterations. }\end{array}$ & I & $\begin{array}{l}\text { Moderate alterations } \\
\text { from activation of } \\
\text { HTPA axis, e.g., } \\
\text { mild tachycardia, } \\
\text { mild hypertension, } \\
\text { moderately high } \\
\text { fasting blood glucose, } \\
\text { alteration of the } \\
\text { eating habits, polyuria. }\end{array}$ & 2 & $\begin{array}{l}\text { Major alterations of activation } \\
\text { of HTPA axis, e.g., tachycardia, } \\
\text { hypertension, high fasting blood } \\
\text { glucose, carbohydrate craving, } \\
\text { bulimia, eating disorders, and } \\
\text { dysuria. }\end{array}$ & 3 \\
\hline
\end{tabular}

Abbreviations: IIBMPE, insomnia induced brief manic-psychotic episodes; HTPA, hypothalamic-pituitary-adrenal axis.

\section{Case no. 3}

This was the case of a 24-year-old female general nurse working night shifts at a local hospital. She reported that she tried to embark also on day shifts to increase her salary thus working in the role of bank nurse. However, due to the accumulation of stress, she spent several weekends by reducing progressively her time of sleep to cover extra shifts at night. Her insomnia ensued gradually while she reported that she felt that she did not need to sleep much to complete her job. Nevertheless, when back to regular and daily shifts, at the beginning of the weeks, her colleagues started to notice some errors in her performance, like clear omissions or incorrect medications administered to patients, minor errors, an unexplainable optimism out of character, and a mild pressure of speech. Besides, she became increasingly suspicious about criticism and remarks from colleagues to the point of believing that her colleagues were against her and that they were jealous of her. She was referred to the occupational health department. The psychiatric team which was involved in the diagnosis believed that this was a case of IIBMPE. The score on the IIBMPE assessment scale was 5 , mostly evidencing minor cognitive errors in her performance and paranoia. All other tests were negative.

\section{Conclusion}

The authors of the current communication have observed a particular form of psychiatric presentation named Insomnia Induced Brief Manic-Psychotic Episodes triggered by prolonged sleep deprivation. The clinical presentation includes specific symptoms with a mixed pattern of hypomanic and behavioural activation with grandiose thoughts, disinhibition, unrealistic expectations, psychosis, suspiciousness, altered concentration, and increased risks for cognitive and procedural errors. Also, there is a complete remission of symptoms after restoring a regular sleep pattern. However, as the current study mostly focussed on the psychiatric presentation of the IIBMPE, the etiopathogenesis model is primarily clinical and applicative. Other theories for explanation need further investigations and are, at the moment, only probabilistic. 


\section{Acknowledgments}

No funds were received by the authors for the current article.

\section{Conflicts of interest}

The authors declare no conflict of interest for the current article. The clinical cases described are not linked to the actual authors' institutions and affiliations.

\section{References}

1. Merriam-Webster Dictionary Online. Insomnia. 2018.

2. Belda X, Armario A. Dopamine D1 and D2 dopamine receptors regulate immobilization stress-induced activation of the hypothalamus-pituitaryadrenal axis. Psychopharmacology (Berl). 2009;206(3):355-365.

3. Hein M, Senterre C, Lanquart JP, et al. Hyperarousal during sleep in untreated primary insomnia sufferers: A polysomnographic study. Psychiatry Res. 2017;253:71-78.
4. Riemann D, Spiegelhalder K, Feige B, et al. The hyperarousal model of insomnia: A review of the concept and its evidence. Sleep Med Rev. 2010;14(1):19-31.

5. Subramaniam M, Abdina E, Shahwan S, et al. Prevalence, correlates and outcomes of insomnia in patients with first episode psychosis from a tertiary psychiatric institution in Singapore. Gen Hosp Psychiatry. 2018;51:15-21.

6. Lazzari C, Shoka A, Basavaraja P, et al. Sleep deprivation in healthcare professionals and medical errors: How to recognize them? Sleep Med Dis Int J. 2018;2(1):00034.

7. Clark I, Landolt HP. Coffee, caffeine, and sleep: A systematic review of epidemiological studies and randomized controlled trials. Sleep Med Rev. 2017;31:70-78.

8. Lambrichts S, Van Oudenhove L, Sienaert P. Antibiotics and mania: A systematic review. J Affect Disord. 2017;219:149-156.

9. Lichstein KL, Payne KL, Soeffing JP, et al. Vitamins and sleep: An exploratory study. Sleep Med. 2007;9(1):27-32. 